\title{
Efficient Calibration of Contact-less Resonant Bio-sensor Affected by Operating Conditions
}

\author{
Anthony Deluthault, Vincent Kerzérho, Serge Bernard and Fabien Soulier \\ Montpellier Laboratory of Informatics, Robotics and Microelectronics \\ University of Montpellier \\ 161 rue Ada 34095 Montpellier Cedex 5, FRANCE \\ contact: name@lirmm.fr
}

\author{
Philippe Cauvet \\ OPHTIMALIA \\ 5 Esplanade Anton Philips \\ Campus EffiScience \\ 14460 Colombelles - France \\ contact: philippe.cauvet@ophtimalia.com
}

\begin{abstract}
In this paper, a new model-based method is presented for the development of calibration technique of inductively coupled biosensors. This method is applied to the case of a lensbased intra-ocular pressure sensor. A new performance indicator is proposed. It is the $2^{n d}$-order coefficient of the single-variable quadratic polynomial regression approximating the Bode phase plot of the system. This new indicator is reliable to significant distance and angle variations between primary and secondary and to small signal-to-noise-ratio.
\end{abstract}

Keywords-Biosensor, Intra-Ocular Pressure, Inductive Coupling, Calibration.

\section{INTRODUCTION}

Implanted and wearable electrical sensors are more and more used for health monitoring [1]. For such kind of application, the sensor must ensure the highest level of safety for the patient. An interesting approach consists in using only passive sensor at the patient interface. In other words, the patient is only in contact with a passive part of the sensor. This passive part avoids any unsafe stand-alone action and because of its low cost they can be discarded after single patient use. In this context, sensors based on magnetic coupling seems very suitable. The idea is that the electrical characteristics of the passive part of the sensor, worn by the patient, is sensitive to the biological parameter to monitor. The modifications of electrical characteristics are seen by the active reader like a load variation modifying the resonance frequency of the system. This kind of system can be used for monitor different physiological parameters: garment pressure on limb [2] , electrocardiogram (ECG) [3], intra-ocular pressure (IOP) [4]. However these sensors are wearable and in direct contact with the human. This means that they are subject to a set of disturbing factors [5] which forces the use of a calibration technique to make them insensitive to these disturbances.

We propose an efficient calibration technique for inductively coupled biosensors. This method is applied to the case study of a lens-based IOP measurement system. The principle of this IOP sensor and the errors induced by operating conditions are described in section II. The calibration process is described in section III. The section IV presents the validation and generalization of the calibration process.

\section{OVERVIEW}

\section{A. Principle of the IOP Sensor}

Developing wearable IOP measurement systems aims at monitoring this physiological parameter over a day. Indeed IOP follows a 24-hour cycle whose variations can be related to glaucoma disease. The figure 1 presents the principle of the system. The first part of the system is a passive sensor embedded in a lens. This is a LC-resonant circuit whose resonance frequency variations are directly related to IOP variations. The resonance frequency is measured through a inductive coupling powered by a primary antenna embedded in the spectacle frame worn by the patient. The relevant data is the resonance frequency of the system. It is measured on the Bode phase plot such as the one of the graphs presented on figure 2. The spectacle frame also embeds some electronics for signal generation and processing. Data are then wirelessly transmitted to a portable device for storage.

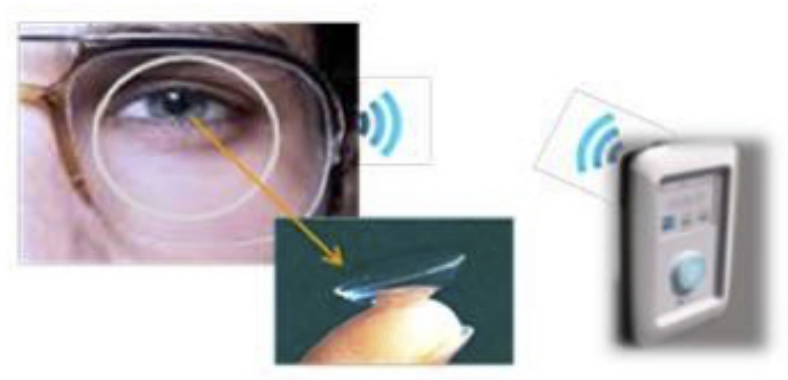

Fig. 1. principle of IOP measurement system

\section{B. Variability in operation}

As such systems as IOP measurement one aim at operating during 24-hour, they become strongly sensitive to a lot of disturbing phenomena potentially affecting the quality of the measurement. Those phenomena [5] can be various as the air humidity, the temperature variation, the eye movement, the eye blinking, the distance between the primary and the secondary or an external electromagnetic field for instance.

The quality of communication or of energy transferring 


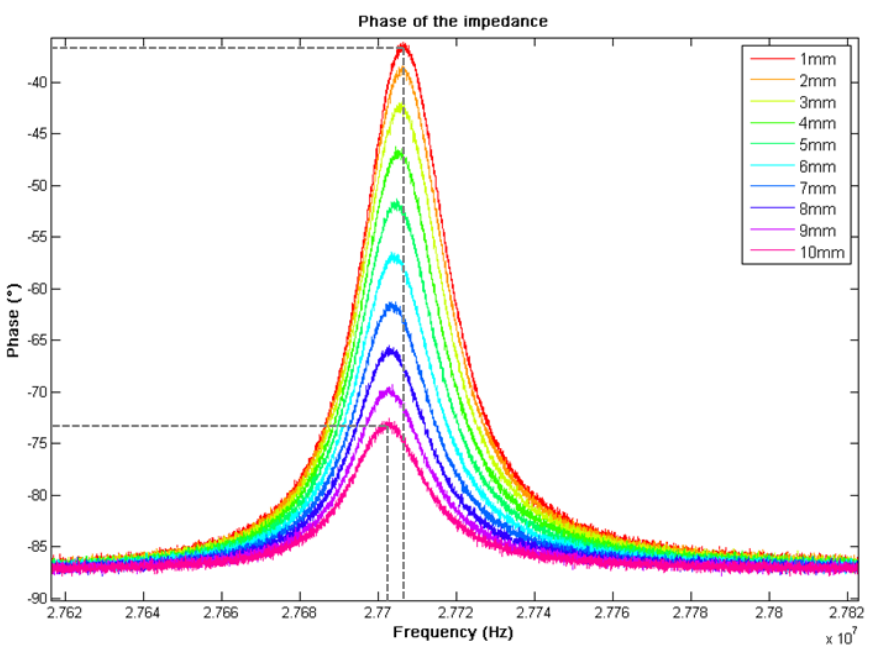

Fig. 2. Bode phase of the system for different distances between primary and secondary

in inductively coupled systems are strongly related to the coupling coefficient $\mathrm{k}$. It depends on several factors such as the shape and the dimensions of the two antennas and the distance and the angle between them.

In our case we will focus on the impact of distance and angle variations on the coupling coefficient that directly impact the resonance frequency observed on the Bode phase plot. Because the system will be worn during 24 hours the distance and the angle will be sources of disturbance as the patient will move the primary on his nose or just turn the eyes.

As we can see in figure 2 , the distance disturbs the phase in frequency and in amplitude. The frequency shift is about around $4.5 \mathrm{kHz}$ which means in $\mathrm{mmHg}$ a variation of around $13.6 \mathrm{mmHg}$ which is significant as regular human IOP varies between 15 and $25 \mathrm{mmHg}$.

\section{Calibration techniques for resonant biosensor}

The impact of varying distance between primary and secondary of resonant biosensor has already been observed by the authors of [6]. They have proposed a calibration technique based on this observation. This calibration technique is based on the observation of a linear correlation between the resonance frequency shift due to distance variation and the amplitude of the Bode phase plot. Their proposed calibration technique is done according to the following process.

At first, the baseline of the phase curve is removed based on the $1^{\text {st }}$-order polynomial regression computed on the whole curve. Then the data points under $10 \%$ of the maximum phase value are removed and a $3^{r d}$-order polynomial is fitted to the remaining data. The resonance frequency is found as the maximum value of the fitted curve. The same method is used to find the local minimum on the left of the resonance frequency. The height of the phase peak is defined as the indicator and it is calculated as the difference between the estimated minimum and maximum phase values. This operation is repeated for different distances. Then the resonance frequency is plotted in function of the height of the phase as presented by figure 3 . Then a $1^{\text {st }}$-order polynomial fitting is computed on this curve.

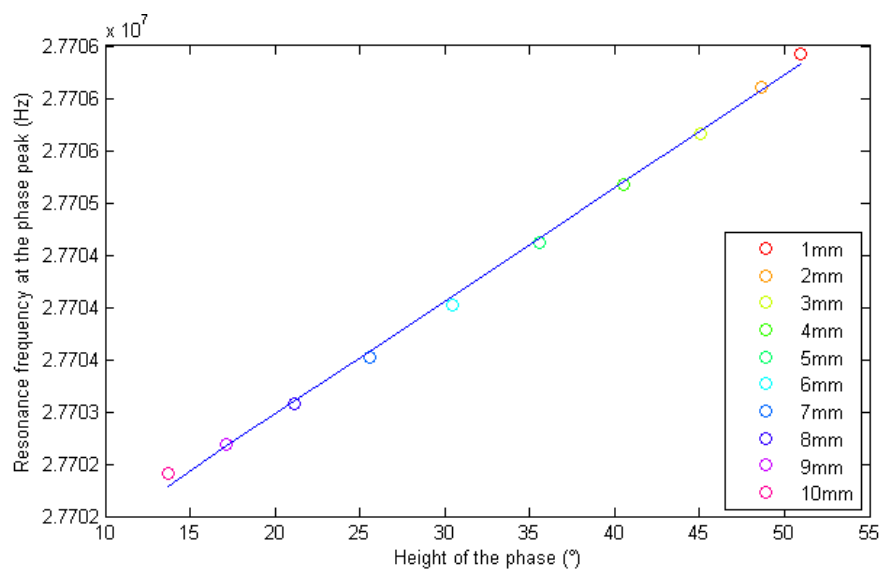

Fig. 3. Resonance frequency at the phase peak in function of the height of the phase

Then the calibration consists in correcting each measurement. Indeed the new measured resonance frequency is corrected by subtracting the product of the corresponding height of the phase and the $1^{\text {st }}$-order coefficient of the polynomial fitting.

Here we want to develop a new calibration strategy, based on a theoretical analysis and reusable for different indicator and type of biosensor. To have a good calibration strategy, it must be based on a pertinent indicator with an intrinsic correlation with the performance. Then it is mandatory to have a precise correlation model to estimate the correction.

\section{PERFORMANCE INDICATOR}

\section{A. introduction}

In order to estimate correctly a correction, a good indicator must be strongly correlated with the performance and also immune to noise. In this part we develop a new strategy based on an theoretical analysis that use a model in order to find a new performance indicator to estimate a correction. We present results that use the indicator from [6] and our new indicator for varying distances between primary and secondary.

\section{B. Modelization}

In order to define an efficient correction technique, we propose an electrical model of the communication between the primary and the secondary. This electrical model is given by the schematic in figure 4 [7].

On this schematic we can see two parts: the antenna and the sensor which are made with RLC elements. The antenna and the sensor are respectively the primary and the secondary of the magnetically-coupled communication system. The first step is to find the total impedance of the circuit which is equation 3. To do this we have to admit there is a voltage $V_{21}$ in the primary induced by the electrical field of the secondary and reciprocally $V_{12}$ in the secondary by the primary equation 1 and 2.

$$
V_{21}=j \omega M(x) I_{2}
$$




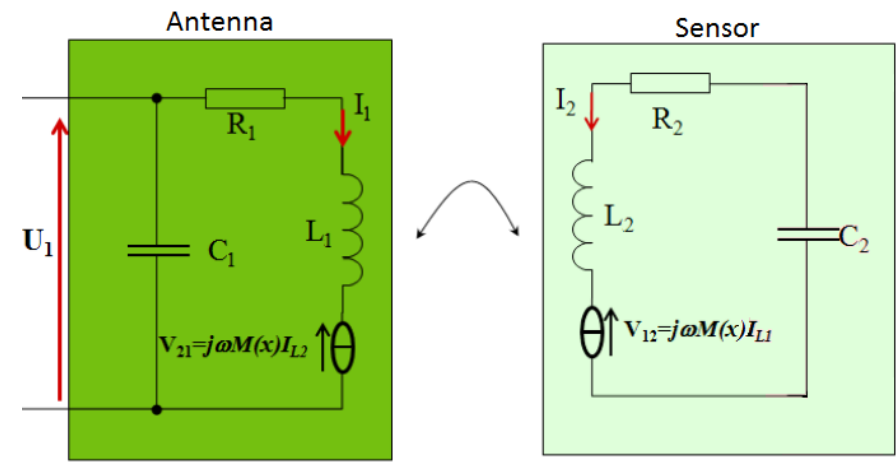

Fig. 4. Electrical model of the inductively coupled system

$$
\begin{gathered}
V_{21}=j \omega M(x) I_{1} \\
Z_{T O T}=R_{1}+j \omega L_{1}+\frac{M^{2}(x) \omega^{2}}{\frac{1}{C_{2} j \omega}+R_{2}+j \omega L_{2}} \\
M(x)=k \sqrt{L_{1} L_{2}}
\end{gathered}
$$

We can see that the mutual inductance $\mathrm{M}$, and consequently the coupling factor $k$, take a role in the equation 3 of the total impedance. This mutual inductance can be calculated with the equation 5 which depends on the distance and the angle between the primary and the secondary.

$$
M(x)=\frac{\mu_{0}}{4 \pi} \oint_{C_{1}} \oint_{C_{2}} \frac{d r \overrightarrow{1} \cdot d r \overrightarrow{2}}{r \overrightarrow{1}-r \overrightarrow{2}}
$$

The mutual inductance can be calculated numerically with the equation 6 [8].

where $\mathrm{r} 1$ and $\mathrm{r} 2$ represent the two loops, defined in Cartesian coordinates as a discretized parametric equation. The mutual inductance is depending on the geometrical of the primary and the secondary. In our case it is calculated with 3 circles as the primary and 4 circles as the secondary. Then we can determinate the characteristic value of each element of the circuit. The characteristics values of the elements of the secondary are taken from the corresponding data-sheet of the sensor. For the primary as we can see in the equation 1 the capacitive element is not taken into account. The inductance of the primary is calculated with the equation 7 [9]

$$
L=\frac{0.3937\left(\frac{r_{i}+r_{o}}{2} N\right)^{2}}{8 \frac{r_{i}+r_{o}}{2}+11\left(r_{o}-r_{i}\right)}(\mu H)
$$

where $r_{i}$ and $r_{o}$ are related to the antenna dimension and $\mathrm{N}$ is the number of turns. Then the image of the impedance is produced in order to obtain the phase presented in figure 2 depending on the distance and the angle.

We will now apply the two calibration techniques. The first technique has been described in section II-C which uses the height of the phase as indicator. The second method is a new one which uses the $2^{\text {nd }}$-order coefficient of the singlevariable quadratic polynomial fit to a part of the phase curve as indicator. We will apply those correction methods for two ranges of distance in order to see their precision and their reliability to noise.

\section{Case of height of phase}

This case shows the result obtained by using the technique and indicator described in section II-C. This method is based on the computation of an indicator based on the difference between estimated maximum and minimum values of the phase curve.

The figure 5 shows that this calibration technique is precise for close range which is 0 to $10 \mathrm{~mm}$ of distance. This method is more precise for close range than our method that is presented in the next section.

\section{Case of the second order coefficient}

In this case, we use a different indicator. The new indicator is based on an observation. Indeed the phase curve close to the resonance frequency describes a parabola curvature. In addition the radius of curvature changes with the distance variation between primary and secondary.

Then the new calibration technique follows this process. At first, the baseline is removed. Then the data points which are taken are the 5\% of the total point number around the local maximum phase found. Then a single-variable quadratic polynomial is fitted to the remaining data and the resonance frequency is found as the maximum value of the fitted curve. The $2^{\text {nd }}$-order coefficient of the fitted curve is used as the indicator correlated with the resonance frequency shift due to distance variation. A calibration distance is selected $(5 \mathrm{~mm}$ for 0 to $10 \mathrm{~mm}$ for example). Then the delta of the resonance frequency is calculated for each distance with the resonance frequency of the calibrated distance. We fit the delta of frequencies in function of the $2^{\text {nd }}$-order coefficients curve and compute a $1^{\text {st }}$-order polynomial fitting on it as presented in figure 6 .

The compensated frequency is found by using a $2^{n d}$-order coefficient found at the measured resonance frequency in the $1^{s t}$-order polynomial and then it is subtracted to the measured resonance frequency. This method is reusable for the angle as the delta of frequencies in function of the $2^{\text {nd }}$ order coefficients curve points stay in the $1^{\text {st }}$-order polynomial when the angle is changed.

The figure 7 shows that the second order coefficient is more robust to the noise than the method from which is out of range for larger distance. Indeed by increasing the distance, the amplitude of the phase curve is reduced. As a consequence the signal-to-noise ratio is also reduced. In the case of the method from [6] by removed the $10 \%$ point of the maximum phase value on small amplitude phase the $3^{\text {rd }}$-order polynomial is made on the whole curve which bias the measured resonance frequency and consequently the correction. 


$$
M(x)=\frac{\mu_{0}}{4 \pi} \sum_{k=1}^{k=N_{1}-1} \sum_{l=1}^{l=N_{2}-1} \frac{\Delta r 1_{x}(k) \Delta r 2_{x}(l)+\Delta r 1_{y}(k) \Delta r 2_{y}(l)+\Delta r 1_{z}(k) \Delta r 2_{z}(l)}{\sqrt{\left(r 1_{x}(k)-r 2_{x}(l)\right)^{2}+\left(r 1_{y}(k)-r 2_{y}(l)\right)^{2}+\left(r 1_{z}(k)-r 2_{z}(l)\right)^{2}}}
$$

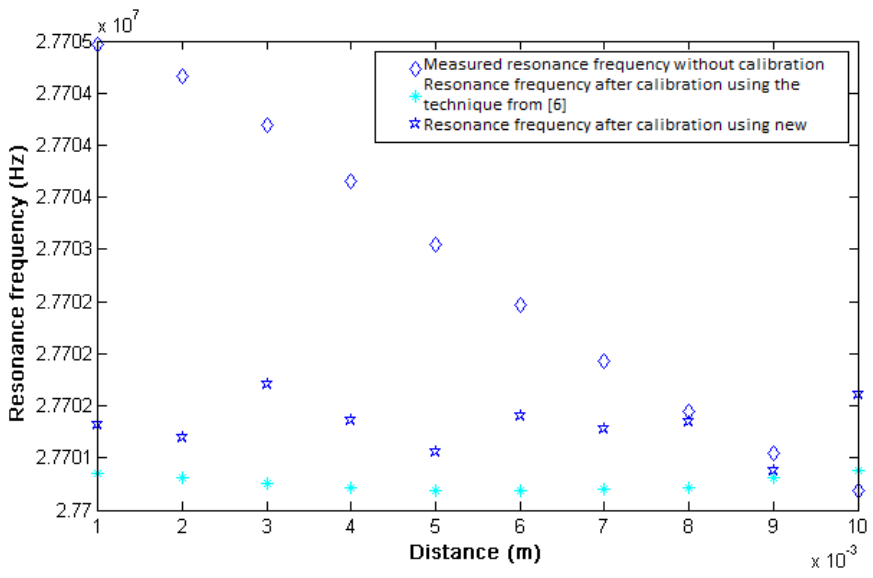

Fig. 5. Variation of resonance frequency measurement for distance variations from 0 to $10 \mathrm{~mm}$

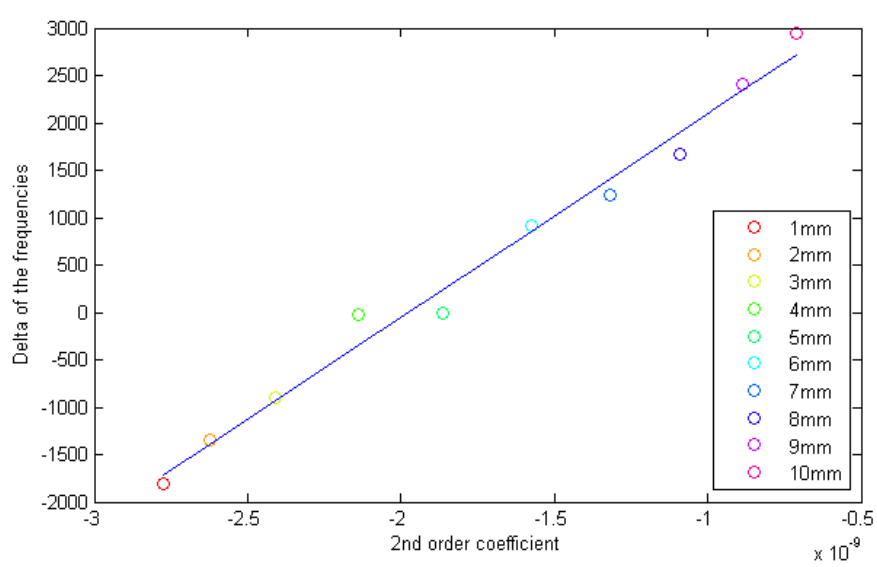

Fig. 6. Delta of the frequencies inf function of the 2 nd order coefficient

\section{NEW CALIBRATION TECHNIQUE VERSUS ANGLE AND DISTANCE VARIATIONS}

In this part, we show the results based on the same model than previously where theoretical analysis have been done for the calibration against distance and angle effects using the $2^{n d_{-}}$ order coefficient as indicator.

The figure 8 shows the measured pressure variation related to the environmental condition variation as the distance and the angle between the primary and the secondary coils. We can see that the error induced by the angle and the distance is close to the normal mean IOP measurement done on a human which is around 15 to $25 \mathrm{mmHg}$.

The figure 9 shows that our correction method is reusable for the angle and induces an error smaller than one $\mathrm{mmHg}$ for the ranges of distance and angle set for the simulation. As we can see on the figure, the correction is less stable for extreme conditions as the $2^{\text {nd }}$ order coefficient and the resonance frequency are harder to find in the noise. This

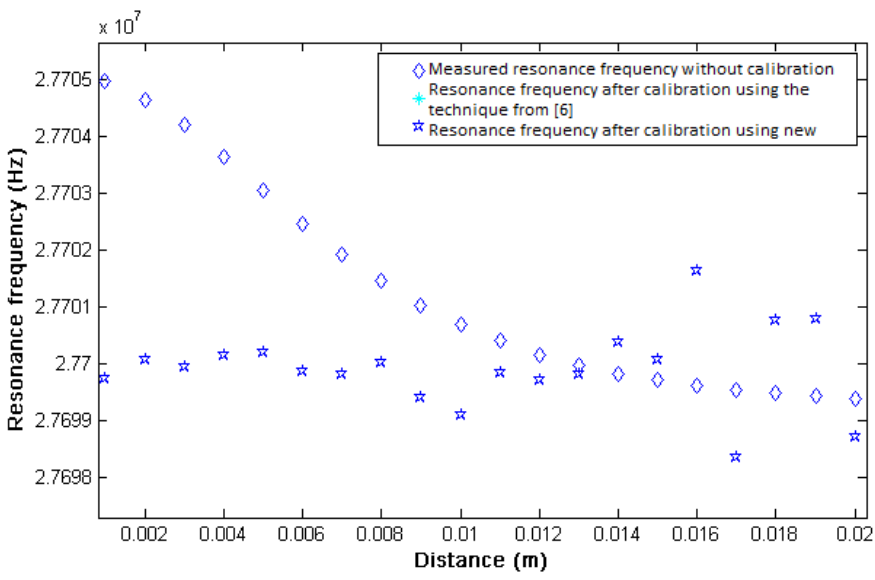

Fig. 7. Variation of resonance frequency measurement for distance variations from 0 to $20 \mathrm{~mm}$

encourage ourselves to do the simulation with more realistic distance like 0 to $30 \mathrm{~mm}$.

\section{DISCUSSION}

We observed that the technique described in [6] is efficient for close range with not too small amplitude phase which restrict it in terms of use case and re-usability. It is more precise than our method which is more robust to the noise with bigger distance and smaller amplitude phase. This lead us to do more simulation with larger distance.

This observation is obviously related to a particular case which is a lens-based IOP sensor which is made of particular set-up and range of operating conditions. All the results are based on simulation, we expect experimental validation of the method. The simulation tool based on the electrical model of the coupling could help finding new indicator of impact of phase and angle variations. For the first described techniques the correction is based on the $1^{\text {st }}$-order interpolation of the curve representing the indicator versus the estimated resonance frequency while for the second described techniques the correction is based on the $1^{\text {st }}$-order interpolation of the curve representing the indicator versus a delta of resonance frequency.Thanks to the simulation tool, it could also be possible to identify more precise correction techniques according to the case study.

\section{CONCLUSION}

To conclude, we have provided a theoretical explanation of the already presented impact of the variation of distance between primary and secondary of a inductively coupled biosensor. In addition this theoretial explanation has demonstrated that the angle variation between primary and secondary can be similarly modeled and corrected.

We proposed a new calibration technique based on singlevariable quadratic polynomial fitting to compute the indicator related to the resonance frequency measurement affected by operating conditions. This indicator is reliable to significant 


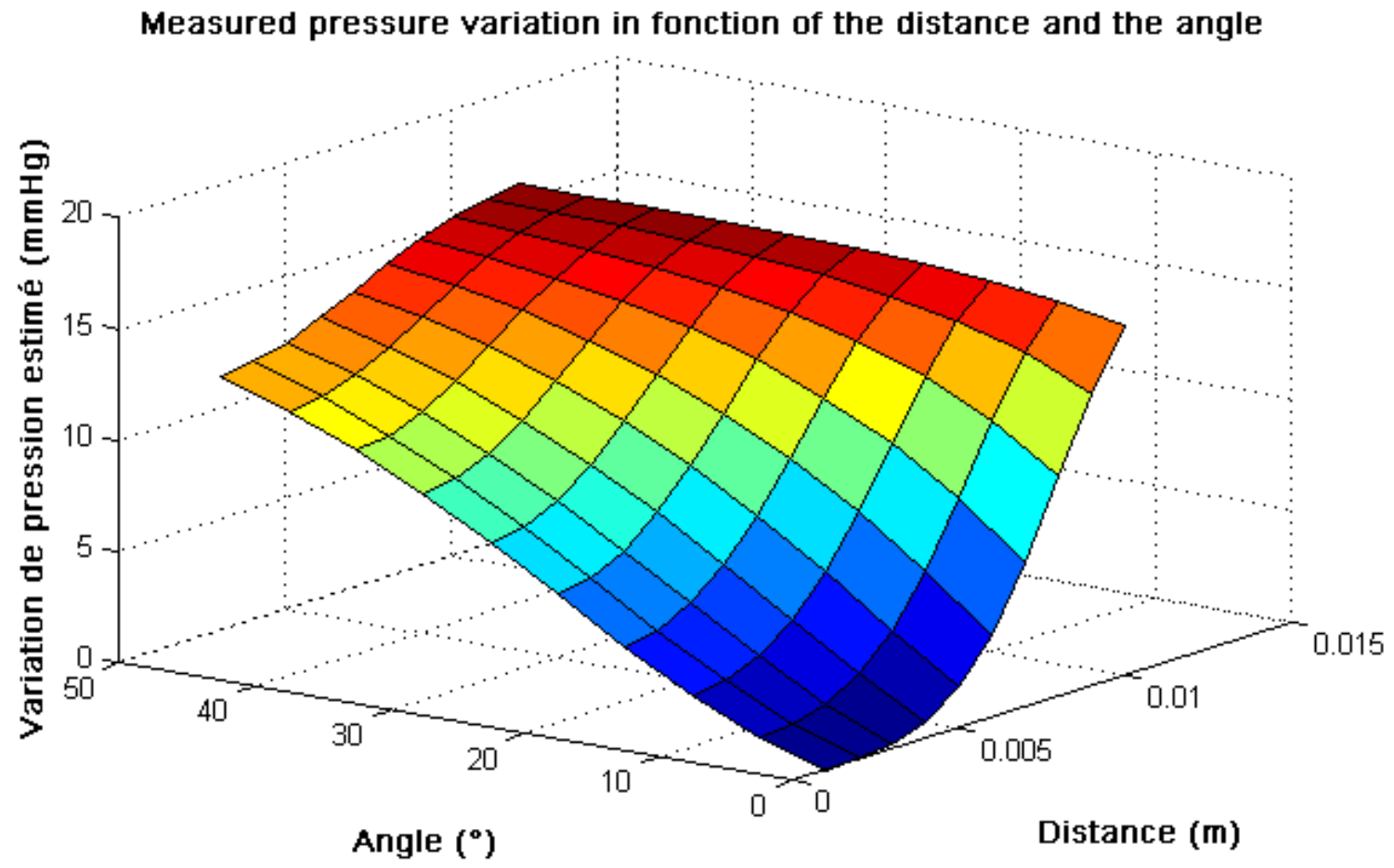

Fig. 8. Measured IOP versus distance and angle before calibration

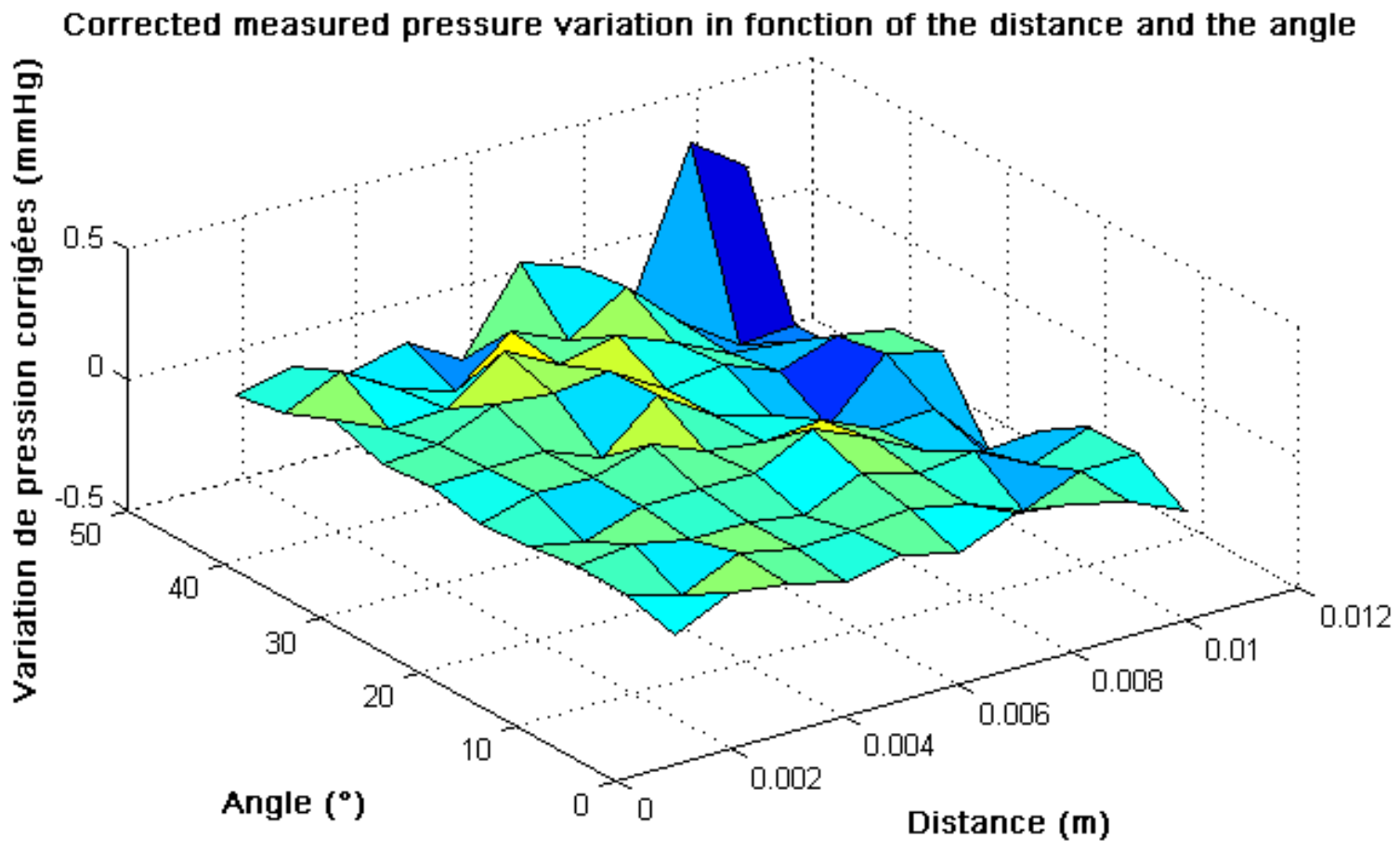

Fig. 9. Measured IOP versus distance and angle after calibration

distance and angle variations (over $10 \mathrm{~mm}$ for distance). These results are strongly related to the case study that defines the distance and angle variations but also the primary and secondary dimensions.

Moreover, as our correction technique definition is based on analytical description of the case study in terms of dimensions of sensor and primary antenna, we can expect that this method can be applied to any case study and can be a tool to help designer to chose the dimensions of the different element of the system as much as the range of operating conditions in terms of distance and angle between primary and secondary. 


\section{ACKNOWLEDGMENT}

This work benefited from the support of the project SACSO ANR of the French National Research Agency (ANR).

\section{REFERENCES}

[1] J. Andreu-Perez, D. Leff, H. Ip, and G.-Z. Yang, "From wearable sensors to smart implants- x2013;toward pervasive and personalized healthcare," Biomedical Engineering, IEEE Transactions on, vol. 62, no. 12, pp. 2750-2762, Dec 2015.

[2] T. Salpavaara, J. Verho, P. Kumpulainen, and J. Lekkala, "Readout methods for an inductively coupled resonance sensor used in pressure garment application," Sensors and Actuators A: Physical, vol. 172, no. 1, pp. 109 - 116, 2011, eurosensors XXIV, Linz, Austria, 5-8 September 2010. [Online]. Available: http://www.sciencedirect.com/science/article/pii/S0924424711001245

[3] J. Riistama, E. Aittokallio, J. Verho, and J. Lekkala, "Totally passive wireless biopotential measurement sensor by utilizing inductively coupled resonance circuits," Sensors and Actuators A: Physical, vol. 157, no. 2, pp. 313 - 321, 2010. [Online]. Available: http://www.sciencedirect.com/science/article/pii/S0924424709005251

[4] P. Auvray, L. Rousseau, G. Lissorgues, F. Soulier, O. Potin, S. Bernard, F. Dieuleveult, E. Scorsone, P. Bergonzo, L. Chicaud, S. Picaud, and J. Sahel, "A passive pressure sensor for continuously measuring the intraocular pressure in glaucomatous patients," $\{$ IRBM $\}$, vol. 33, no. 2, pp. 117 - 122, 2012, numro spcial $\{$ ANR \{TECSANTechnologie\} pour la sant et l'autonomie. [Online]. Available: http://www.sciencedirect.com/science/article/pii/S1959031812000036

[5] M. Andraud, A. Deluthault, M. Dieng, F. Azas, S. Bernard, P. Cauvet, M. Comte, T. Kervaon, V. Kerzrho, S. Mir, P. H. Pugliesi-Conti, M. Renovell, F. Soulier, E. Simeu, and H. G. Stratigopoulos, "Solutions for the self-adaptation of communicating systems in operation," in $\mathrm{On}$ Line Testing Symposium (IOLTS), 2014 IEEE 20th International, July 2014, pp. 234-239.

[6] T. Salpavaara, J. Verho, P. Kumpulainen, and J. Lekkala, "Wireless interrogation techniques for sensors utilizing inductively coupled resonance circuits," Procedia Engineering, vol. 5, pp. $216-219,2010$, eurosensor $\{$ XXIV $\}$ ConferenceEurosensor $\{\mathrm{XXIV}\}$ Conference. [Online]. Available: http://www.sciencedirect.com/science/article/pii/S1877705810006338

[7] M. Dieng, F. Azas, M. Comte, S. Bernard, V. Kerzrho, M. Renovell, T. Kervaon, and P. H. Pugliesi-Conti, "Study of adaptive tuning strategies for near field communication (nfc) transmitter module," in Mixed-Signals, Sensors and Systems Test Workshop (IMS3TW), 2014 19th International, Sept 2014, pp. 1-6.

[8] A. Vena and P. Roux, "Near field coupling with small frid objects,", in Progress In Electromagnetic Research Symposium Proceedings, Moscow, Rassia, August 2009.

[9] Y.Lee, "Antenna circuit design for rfid applications," Microchip Technology, Tech. Rep., 2003. 